\title{
Mitochondria-mediated damage to dopaminergic neurons in Parkinson's disease (Review)
}

\author{
XIAO-LIANG LIU ${ }^{1}$, YING-DI WANG ${ }^{2}$, XIU-MING YU ${ }^{3}$, DA-WEI LI ${ }^{4}$ and GUANG-REN LI ${ }^{5}$ \\ ${ }^{1}$ Cancer Center, The First Hospital of Jilin University, Changchun, Jilin 132021; ${ }^{2}$ Department of Urinary Surgery, \\ The Tumor Hospital of Jilin Province, Changchun, Jilin 130012; ${ }^{3}$ Department of Immunology, The First Affiliated Hospital \\ to Changchun University of Chinese Medicine, Changchun, Jilin 130021; ${ }^{4}$ Department of Neurology, Affiliated Hospital \\ of Beihua University, Jilin, Jilin 132000; ${ }^{5}$ Department of Neurology, The Third Hospital of Jilin University, \\ Changchun, Jilin 130021, P.R. China
}

Received January 22, 2016; Accepted October 20, 2017

DOI: $10.3892 /$ ijmm.2017.3255

\begin{abstract}
Mitochondria are important organelles in virtually all eukaryotic cells, and are involved in a wide range of physiological and pathophysiological processes. Besides the generation of cellular energy in the form of adenosine triphosphate, mitochondria are also involved in calcium homeostasis, reactive oxygen species production and the activation of the intrinsic cell death pathway, thus determining cell survival and death. Mitochondrial abnormalities have been implicated in a wide range of disorders, including neurodegenerative disease such as Parkinson's disease (PD), and considered as a primary cause and central event responsible for the progressive loss of dopaminergic neurons in PD. Thus, reversion or attenuation of mitochondrial dysfunction should alleviate the severity or progression of the disease. The present review systematically summarizes the possible mechanisms associated with mitochondria-mediated dopaminergic neuron damage in $\mathrm{PD}$, in an attempt to elucidate the requirement for further studies for the development of effective PD treatments.
\end{abstract}

Correspondence to: Professor Guang-Ren Li, Department of Neurology, The Third Hospital of Jilin University, 126 Xiantai Street, Changchun, Jilin 130033, P.R. China

E-mail: lgren18@hotmail.com

Abbreviations: PD, Parkinson's disease; SNpc, substantia nigra pars compacta; ATP, adenosine triphosphate; ROS, reactive oxygen species; OMM, outer mitochondrial membrane; IMM, inner mitochondrial membrane; VDAC, voltage-dependent anion channel; ETC, electron transport chain; $\mathrm{MCU}$, mitochondrial $\mathrm{Ca}^{2+}$ uniporter; ANT, adenine nucleotide translocator; $\mathrm{mPTP}$, mitochondrial permeability transition pore; HNE, 4-hydroxyl-2-nonenal; SN, substantia nigra; mtDNA, mitochondrial DNA; MPTP, 1-methyl-4-phenyl-1,2,3,6-tetrahydropyridine; VMAT2, vesicular monoamine transporter 2; OPA1, optic atrophy protein 1

Key words: Parkinson's disease, pathogenesis, mitochondrial dysfunction, reactive oxygen species

\section{Contents}

1. Introduction

2. Mitochondrial structure and function

3. Mitochondrial ROS production and vulnerability of dopaminergic neurons to oxidative damage

4. Mitochondria $\mathrm{Ca}^{2+}$-induced cell damage

5. mtDNA deletions in PD

6. Abnormalities of mitochondrial fusion and fission in PD

7. Conclusion

\section{Introduction}

Parkinson's disease (PD) is a common neurodegenerative disorder, characterized by the progressive degeneration and death of dopaminergic neurons, and by the expression of Lewy bodies in the surviving neurons of the substantia nigra (SN) (1). The exact mechanism of dopaminergic neuron damage remains poorly understood, but several lines of evidence implicate mitochondrial dysfunction as a possible primary cause for the cell death in PD (2-5). Mitochondria are pleiotropic organelles affecting a wide range of molecular and cellular processes. Besides generation of cellular energy in the form of adenosine triphosphate (ATP) by oxidative phosphorylation, they play a crucial role in calcium homeostasis, reactive oxygen species (ROS) production and intrinsic cell death pathway regulation, thus determining cell survival and death $(2,5,6)$. Mitochondria are therefore vital for normal cellular function, including intracellular metabolic activities and signal transduction of various cellular pathways. Mitochondrial dysfunction has been considered as a central event and primary initiator responsible for the progressive loss of dopaminergic neurons in PD (6-8). A better understanding of the molecular mechanisms underlying the pathogenesis will provide potential targets acting as blocking or reversing factors to limit PD progression.

\section{Mitochondrial structure and function}

Mitochondria are important cytoplasmic organelles with double lipid membranes: The outer mitochondrial membrane (OMM) and the inner mitochondrial membrane (IMM). The elaborate 
structures of the mitochondria are required for the maintenance of their normal functions (9). The OMM contains numerous channels formed by the protein, which allow the passage of ions and low molecular-weight substances from the relatively permeable membrane (10). The voltage-dependent anion channel (VDAC) is essential for the exchange of metabolites between the cytosol and intermembrane space bordered by the OMM and the IMM (11). A large hydrophilic pore of the VDAC provides a structural device for the translocation of ions and a variety of metabolites, including ATP and adenosine diphosphate (12). The OMM is involved in mitochondria-mediated cell death via various different pathways through the interaction with a number of regulator proteins. Bcl-2-associated X protein (Bax), for example, translocates from the cytosol into the OMM in response to the apoptotic signals, subsequently oligomerizing at the OMM and promoting the release of apoptotic factors cytochrome $c$ and apoptosis-inducing factor, and other pro-apoptotic mediators (13).

The IMM is a convoluted structure formed by a large number of infoldings known as mitochondrial cristae, and is almost impermeable, thus providing an efficient barrier to form an electron gradient and a relatively closed inner matrix for the electron transport chain (ETC), which is required for oxidative phosphorylation in ATP formation (14). The specialized cation transporters and exchangers mediate the cation transmembrane fluxes that are essential for the maintenance of mitochondrial bioactivities. Mitochondrial $\mathrm{Ca}^{2+}$ uniporter (MCU) is a specific transport system for $\mathrm{Ca}^{2+}$ intake across the IMM, thus playing a role in buffering cytosolic $\mathrm{Ca}^{2+}$. The maintenance of physiologically relevant free $\mathrm{Ca}^{2+}$ is required for normal mitochondrial functions, but overload contributes to the opening of the mitochondrial permeability transition pore (mPTP) and matrix swelling, and subsequently cell death (15). The extrusion of mitochondrial $\mathrm{Ca}^{2+}$ depends mainly on the $\mathrm{Na}^{+}$-dependent $\mathrm{Na}^{+}-\mathrm{Ca}^{2+}$ exchanger and the $\mathrm{H}^{+}-\mathrm{Ca}^{2+}$ exchanger $(16,17)$. The $\mathrm{Na}^{+}-\mathrm{H}^{+}$exchanger is a mitochondrial channel that contributes to the maintenance of intracellular $\mathrm{pH}$, which is required for mitochondrial membrane potential formation (14).

The mPTP is a transmembrane channel formed at the contact sites between the OMM and the IMM. Although the components of the MPTP remain controversial, the VDAC, the adenine nucleotide translocator (ANT) and cyclophilin D (CyPD) appear to be implicated $(18,19)$. The mitochondrial matrix protein CyPD mediates direct connection of the IMM protein ANT with the VDAC by binding to a proline residue in ANT (20). The binding results in a conformation of ANT that converts it into a non-specific pore (21). Mitochondrial $\mathrm{Ca}^{2+}$ overload and excessive ROS production may be key inducers in the translocation of CyPD from the matrix to IMM, since each plays a crucial role in mPTP opening (13).

The ETC is a multisubunit complex that is required for the production of ATP via oxidative phosphorylation. Synthesis of ETC proteins depends on mitochondrial DNA (mtDNA) and nuclear DNA. mtDNA is double stranded and encodes for 22 transfer RNAs (tRNAs), 2 ribosomal RNAs and 13 polypeptides that are all subunits of respiratory chain complexes (22). Energy production is the most important function for mitochondria, and mitochondrial ATP is generated via oxidative phosphorylation within the IMM (23). Mitochondria are a major source of ROS, which are produced at the sites of the
ETC (24,25). During oxidative phosphorylation, the respiratory chain complexes I and III leak electrons to oxygen, producing primarily superoxide radicals, and subsequently hydrogen peroxide $\left(\mathrm{H}_{2} \mathrm{O}_{2}\right)$ and hydroxyl radicals $(26,27)$. Overall, mitochondria participate in energy metabolism, ROS production, calcium homeostasis, the stress response and programmed cell death, thus determining cell survival and death.

\section{Mitochondrial ROS production and vulnerability of dopaminergic neurons to oxidative damage}

Mitochondria are primary intracellular source of ROS that are generated from the interaction of unpaired electrons with molecular $\mathrm{O}_{2}$ during oxidative phosphorylation $(28,29)$. Respiratory chain complexes I and III are the major sites of ROS production $(26,30,31)$. The first generated ROS is $\mathrm{O}_{2}^{-}$, an amphibolic radical that cannot easily pass through biological membranes and is converted by the mitochondrial matrix enzyme to form $\mathrm{H}_{2} \mathrm{O}_{2}$ in the mitochondrial intermembrane space and cytosol (32). $\mathrm{H}_{2} \mathrm{O}_{2}$ is a stable molecule that can diffuse from the mitochondria into the cellular cytosol and nucleus, where it can be detoxified by glutathione peroxidase and catalase into water (33). However, when the balance of $\mathrm{H}_{2} \mathrm{O}_{2}$ production and antioxidant defense is perturbed, excessive $\mathrm{H}_{2} \mathrm{O}_{2}$ is accumulated and leads to oxidative stress (34). Particularly in the presence of reduced metals ferrous iron $\left(\mathrm{Fe}^{2+}\right)$, via the Fenton reaction, $\mathrm{H}_{2} \mathrm{O}_{2}$ can easily be converted into the highly reactive hydroxyl radical, causing further oxidative damage (35). It is widely accepted that complex I inhibition is a leading cause of increased ROS formation (34). This production of ROS damages in turn the components of the respiratory chain, particularly complex I, leading to its further inhibition and more ROS production. The vicious circle formed between mitochondrial impairment and oxidative stress has been implicated in PD pathogenesis, and may cause the progressive degeneration of dopaminergic neurons by triggering sequential downstream events in neurodegenerative conditions $(34,36,37)$. Nigral dopaminergic neurons are frequently exposed to oxidative stress as they contain high levels of lipids and iron, and exhibit increased dopamine metabolism. Consequently, gradual damage to the dopaminergic neurons occurs due to attack by more ROS (37). Accumulating evidence supports the link of oxidative damage and degeneration of dopaminergic neurons in PD pathogenesis (38-41). Studies in postmortem brains of patients with PD have shown increased levels of lipid oxidation product 4-hydroxyl-2-nonenal (HNE), DNA and RNA oxidation products 8-hydroxy-deoxyguanosine and 8-hydroxy-guanosine, and carbonyl modifications of soluble proteins, supporting the involvement of oxidative stress in dopaminergic neuron damage $(37,42)$. The neurotoxins 1-methyl-4-phenyl-1,2,3,6-tetrahydropyridine (MPTP), rotenone and 6-hydroxydopamine are well-known parkinsonism inducers that cause oxidative stress and dopaminergic neuron degeneration in animal models, further supporting the contribution of oxidative stress to PD pathogenesis (43-47). ROS play a major role in causing oxidative stress and damage to all macromolecules, including lipids, proteins and DNA (48).

Lipids participate in membrane fluidity and permeability, and mediate inflammatory processes and apoptotic signals, and 
oxidation is the mechanism responsible for the cell damage and death (49). The brain contains high levels of lipids, particular polyunsaturated fatty acids, which are the most prone to lipid peroxidation and responsible for the susceptibility of the organ to oxidative damage (50). The exposure of polyunsaturated fatty acids to free radicals leads to lipid peroxidation which causes the structural damage of membranes, compromising their integrity and consequently cell viability (51). HNE is one of the most important lipid peroxidation products, and has been considered as a inducer responsible for apoptotic cell death via activation of the caspase cascade and induction of DNA fragmentation (52). HNE can also exacerbate oxidative damage by decreasing the levels of glutathione, the major nonenzymatic antioxidant in the central nervous system (53). The elevated levels of lipid peroxidation product HNE have been detected in the SN as well as the cerebrospinal fluid of PD patients, supporting the reinforcement of the hypothesis that peroxidation of polyunsaturated fatty acids leads to dopaminergic neuron damage in oxidative conditions $(40,54)$.

Nigral dopaminergic neurons are particularly exposed to the increased levels of oxidative stress due to increased dopamine metabolism (55). Dopamine is an unstable molecule that can easily undergo oxidative metabolism to form dopamine quinones and free radicals. Normally, the released dopamine is rapidly sequestered into vesicles via vesicular monoamine transporter 2 (VMAT2). A defect in synaptic vesicle formation or function leads to cytoplasmic accumulation of dopamine (56). Inhibition of VMAT2 causes a sustained increase in the formation of dopamine autoxidation products in the cytoplasm, which reinforces the oxidative damage of dopaminergic neurons (56). The levels of dopamine are regulated by monoamine oxidase (MAO)-A and MAO-B, and the latter appears to be a predominant enzyme to metabolize dopamine in neuronal degeneration conditions $(57,58)$. The metabolism of dopamine mediated by MAO-B produces $\mathrm{H}_{2} \mathrm{O}_{2}$ that diffuses into neighboring dopaminergic neurons where it can react with $\mathrm{Fe}^{2+}$ to form hydroxyl radical, leading to further damage to the neurons $(59,60)$. The product of dopamine quinones, aminochrome, can also contribute to neurodegeneration by promoting superoxide generation, $\alpha$-synuclein fibrillization formation and the neuroinflammatory response $(61,62)$.

Iron can also promote ROS generation and trigger neurotoxicity in neurodegenerative conditions $(63,64)$. As a cofactor for proteins, it is distributed widely in neuronal tissue, particularly the SN (65). However, with aging and degenerative processes such as PD, there is an abnormal, progressive deposition of iron and an increased free iron concentration in the $\mathrm{SN}$ pars compacta (SNpc) (66). The increased levels of iron and hyroxyl radicals have been detected in the $\mathrm{SN}$ of $\mathrm{PD}$ animal models, while total glutathione (glutathione and glutathione disulfide) levels were decreased (67). Administration of the iron chelator desferrioxamine significantly lowers iron levels in the brain and protects against iron and MPTP-induced neurodegeneration in PD mouse models (68). These findings suggest that iron is involved in the process of dopaminergic neuron degeneration in PD. Little is known regarding whether an elevated iron level is a cause or the consequence of neuron damage. However, it is widely accepted that iron-induced toxicity is at least partly responsible for the neuronal cell damage associated with PD (67-69).
Several recent lines of evidence have also implicated reactive nitrogen species in dopaminergic neuronal damage leading to $\mathrm{PD}$ pathogenesis (70). Peroxynitrite $\left(\mathrm{ONOO}^{-}\right)$is a nitrating agent that acts as a strong oxidant and can damage numerous cellular structures and alter their function, leading to cell death (70). High levels of $\mathrm{ONOO}^{-}$result in oxidative damage of mitochondrial lipid and protein, inhibition of ETC, $\mathrm{Ca}^{2+}$ overload, and subsequent mPTP opening and mitochondria-related pro-apoptotic mediator release $(70,71)$.

Taken together, these results show that dopaminergic neurons in the SNpc are frequently subjected to oxidative damage due to their high levels of lipids and iron, as well as increased dopamine metabolism. Damage to the mitochondrial complexes and the subsequent increase in ROS production are considered to be central causative events responsible for the loss of dopaminergic neurons in PD pathogenesis under neurogenerative conditions $(72,73)$. Overproduction of ROS causes them to attack macromolecules such as mtDNA and components of the ETC, and causes mtDNA strand breaks, ETC damage and mitochondrial $\mathrm{Ca}^{2+}$ overloading, leading to further production of ROS. The vicious circle between ROS production and $\mathrm{Ca}^{2+}$ overload favors the sustained opening of mPTP by activation of CyPD (13). Generally, CyPD is a mitochondrial matrix protein that can be translocated to the IMM in response to stimuli. Once located in the IMM, this protein interacts with ANT and changes its conformation, which results in the binding of ANT to VDAC and subsequently mPTP opening (21). Bcl-2 family proteins such as Bax and Bcl-2 homologous killer can also facilitate the opening of the mPTP by translocating and oligomerizing into the OMM as a consequence of oxidative stress (74). The opening of the MPTP causes the collapse of mitochondrial membrane potential and the release of pro-apoptotic mediators from the mitochondria into the cytosol, and subsequently cell death (75) (Fig. 1).

Damage to the ETC and subsequent ROS production form a positive feedback circle that may be a central event driving the progressive loss of dopaminergic neurons under neurodegenerative conditions. Therefore, restoring the function of the ETC and inhibition of ROS production may be a promising method for PD treatment. Coenzyme Q10 (CoQ10), for example, functioning as an endogenous co-enzyme of ETC proteins and an ROS scavenger, plays a crucial role in maintaining the integrity of mitochondrial respiration and the clearance of free radicals (76). In vitro and in vivo studies have shown that CoQ10 inhibits paraquat-, rotenone- and MPTP-induced mitochondrial dysfunction, and subsequently, ROS production (77-79). As an essential cofactor in the mitochondrial ETC and a ROS scavenger, CoQ10, is currently being investigated in clinical trials of PD.

\section{Mitochondria $\mathrm{Ca}^{2+}$-induced cell damage}

$\mathrm{Ca}^{2+}$ is one of important ions required for the cells to maintain their biological functions, and it is involved in the regulation of not only cell survival, but also cell death, in response to a variety of cellular signals $(80,81)$. Mitochondria are crucial organelles in the regulation of cytoplasmic $\mathrm{Ca}^{2+}$ levels and in the maintenance of a $\mathrm{Ca}^{2+}$ concentration gradient across their membrane (82). The driving force for the concentration gradient depends on the ETC, which establishes an electrochemical gradient across the IMM 


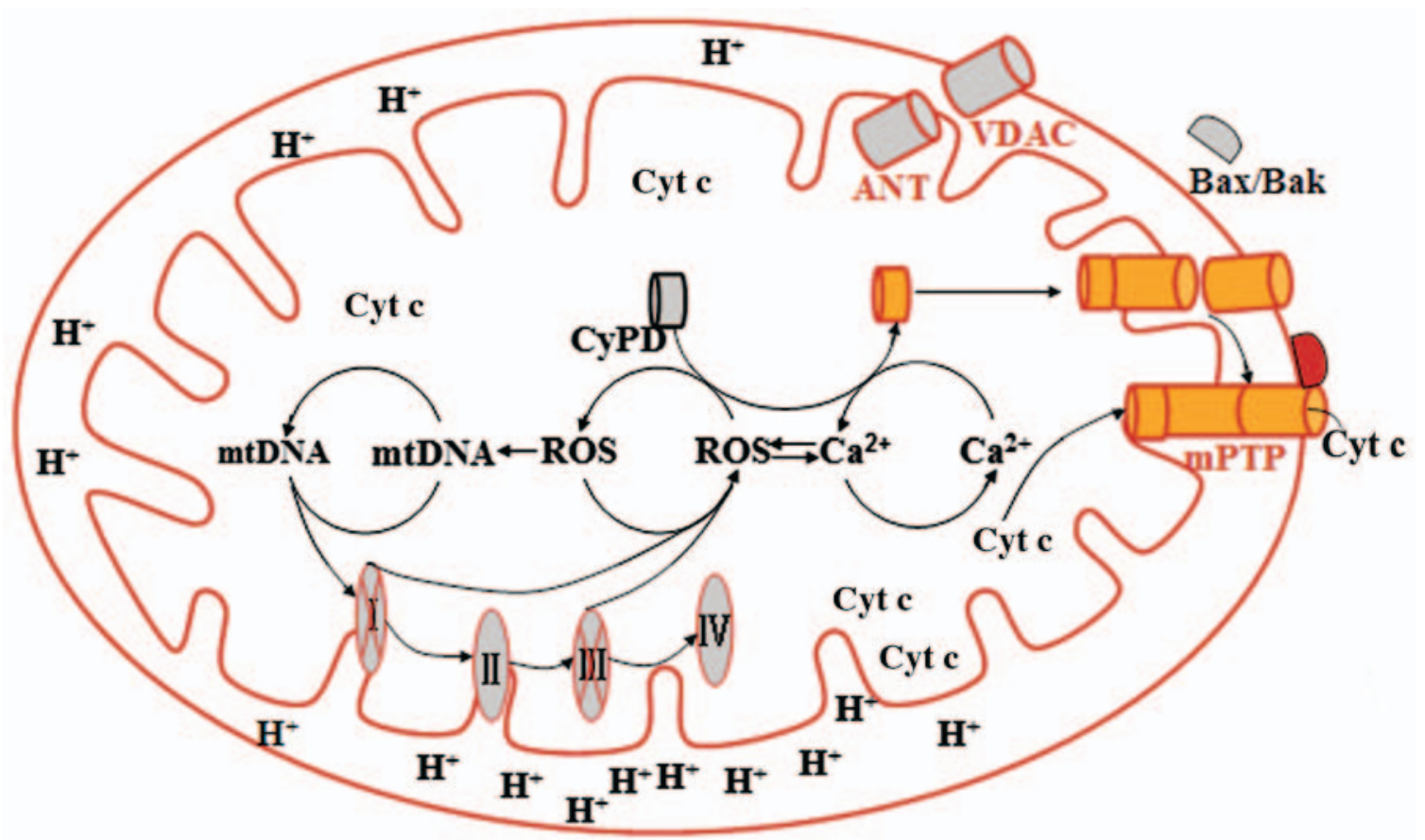

Figure 1. Mitochondrial ROS formation and regulation in apoptosis. Mitochondrial ETC inhibition promotes ROS production. The ROS attack mtDNA and components of the ETC, and cause mtDNA strand breaks, ETC damage and mitochondrial $\mathrm{Ca}^{2+}$ overloading, leading to further production of ROS Mitochondrial ROS and $\mathrm{Ca}^{2+}$ can activate CyPD, a mitochondrial matrix protein that can be translocated to the IMM in response to stimuli. Once located in the IMM, this protein interacts with ANT and changes its conformation resulting in the binding of ANT to VDAC and subsequently mPTP opening. Bcl-2 family proteins such as Bax and Bak can also facilitate the opening of MPTP by translocating and oligomerizing into the OMM as a consequence of oxidative stress. The opening of mPTP causes the collapse of mitochontrial membrane potential and the release of pro-apoptotic mediators such as cytochrome $c$ from the mitochondria into the cytosol. ROS, reactive oxygen species; mtDNA, mitochondrial DNA; ETC, electron transport chain; CyPD, cyclophilin D; IMM inner mitochondrial membrane; OMM, outer mitochondrial membrane; ANT, adenine nucleotide translocator; VDAC, voltage-dependent anion channel; MCU, mitochondrial $\mathrm{Ca}^{2+}$ uniporter; Bax, Bcl-2-associated X protein; Bak, Bcl-2 homologous killer; mPTP, mitochondrial permeability transition pore.

by translocating $\mathrm{H}^{+}$ions to the intermembrane space, thereby generating a membrane potential difference for the entry of $\mathrm{Ca}^{2+}$ into the mitochondria (83). The transportation of cytosolic $\mathrm{Ca}^{2+}$ transports from the OMM into intermembrane space is mediated by the VDAC, which is permeable to $\mathrm{Ca}^{2+}(84) . \mathrm{Ca}^{2+}$ accumulation in the mitochondrial matrix requires the crossing of the relatively impermeable IMM, and the specific transport system, the MCU, is involved (85). The MCU is a two transmembrane domain channel that moves calcium ions across the IMM, and the process is modulated by its regulatory subunits, mitochondrial calcium uptake 1 (MICU1) and MICU2 $(86,87) . \mathrm{Ca}^{2+}$ plays a crucial role in mitochondrial metabolism through the activation of numerous mitochondrial enzymes $(88,89)$. However, mitochondrial $\mathrm{Ca}^{2+}$ overloading may have profound consequences for the cell such as promoting the production of ROS, activating the opening of MPTP and initiating mitochondria-mediated cell death (15). It has been suggested that $\mathrm{Ca}^{2+}$ stimulates the activity of nitric oxide synthase to generate $\mathrm{NO}^{\circ}$, which results in an inhibition of ETC and subsequent ROS production $(90,91)$. NO ${ }^{\bullet}$ can react with $\mathrm{O}_{2}{ }^{--}$to produce $\mathrm{ONOO}^{-}$, a highly reactive radical that causes further damage to the ETC and more ROS production, leading to mitochondrial lipid and protein oxidation, mitochondrial membrane rupture, ATP synthesis decrease and the loss of ion homeostasis (13). Impairment of the ETC increases the production of ROS, which in turn damage components of the respiratory chain, thereby triggering a vicious circle between $\mathrm{Ca}^{2+}$ overload and oxidative stress, leading to irreversible mPTP opening $(92,93) . \mathrm{Ca}^{2+}$ overload favors the sustained opening of the MPTP, which causes the collapse of mitochondrial membrane potential and the swelling of the mitochondria, leading to the release of pro-apoptotic mediators from the mitochondria into the cytosol (94-96) (Fig. 2). Normal neurons are dependent on a calcium signal to regulate diverse processes, including neurotransmitter release, vesicle recycling, axonal transport and neuronal plasticity (97). This is particularly important in dopaminergic neurons that are exposed to frequent influxes of calcium (98). As a central event in mitochondria-mediated intrinsic cell apoptosis, mPTP opening has been reported to be implicated in the pathogenesis of neurodegenerative disorders, including PD $(44,99)$. The MPTP pathway of cell death is mediated by the disruption of the mitochondrial membrane and the release of apoptogenic molecules from the intermembrane space into the cytosol. Cytochrome $c$ is one of the important pro-apoptotic factors released by the mitochondria, which triggers the activation of the caspase cascade, leading to the irreversible process toward apoptosis $(100,101)$. Several studies have reported the inhibition of mitochondrial $\mathrm{Ca}^{2+}$ overload and $\mathrm{mPTP}$ opening, reducing the cell damage in the pathological conditions, including oxidative stress and mitochondrial dysfunction (102). Thus, blockage of mitochondrial $\mathrm{Ca}^{2+}$ overload may attenuate the severity of PD that is closely associated with mPTP opening.

\section{5. mtDNA deletions in PD}

The most important function of the mitochondria is the generation of ATP through the process of oxidative phosphorylation, 




Figure 2. Transporting systems of $\mathrm{Ca}^{2+}$ in mitochondria and regulation of mitochondrial $\mathrm{Ca}^{2+}$ in apoptosis. The difference membrane potential acrossing the IMM forming by translocating $\mathrm{H}^{+}$ions to the intermembrane space drives cytosolic $\mathrm{Ca}^{2+}$ from intermembrane space into mitochondria via MCU. The extrusion of mitochondrial $\mathrm{Ca}^{2+}$ depends mainly on an $\mathrm{Na}^{+}$-dependent $\mathrm{Na}^{+}-\mathrm{Ca}^{2+}$ exchanger and an $\mathrm{H}^{+}-\mathrm{Ca}^{2+}$ exchanger. $\mathrm{Ca}^{2+}$ stimulates activity of nitric oxide synthase to generate $\mathrm{NO}^{\circ}$ and subsequently $\mathrm{ONOO}^{-}$, which can damage ETC, thus promoting the production of ROS, leading to further $\mathrm{Ca}^{2+}$ overloading and ROS production. The overload of mitochondrial $\mathrm{Ca}^{2+}$ activates the opening of $\mathrm{mPTP}$, thus initiating mitochondria-mediated cell death. OMM, outer mitochondrial membrane; IMM, inner mitochondrial membrane; VDAC, voltage-dependent anion channel; $\mathrm{ONOO}^{-}$, peroxynitrite; ETC, electron transport chain; ROS, reactive oxygen species; $\mathrm{MCU}$, mitochondrial $\mathrm{Ca}^{2+}$ uniporter.

which depends on mtDNA-encoded proteins. mtDNA encodes 13 proteins that are all ETC complex subunits involved in ATP production, and 2 RNA and 22 tRNAs required for mitochondrial protein synthesis (103). Due to the proximity to the ETC complexes and the source of ROS release, mtDNA is frequently exposed to oxidative stress (104). The lack of histone protein protection results in mtDNA mutation at a high rate relative to nuclear NDA, particularly in cells with high energy demands (105). A number of mitochondria-related diseases could be linked to mutations of the mitochondrial genome $(104,106-108)$. mtDNA deletions that are closely associated with the deficits in normal mitochondrial activity were previously shown to accumulate in nigral dopaminergic neurons of aged individuals and sporadic PD subjects (106-108). Knockout of the mtDNA transcription gene inhibits the expression of mtDNA and the activities in the respiratory chain of dopaminergic neurons, accompanied with progressive parkinsonism in mouse models (109). High levels of mtDNA deletions in the brains of retenone-induced PD models were observed in the midbrain, but not in the cortex (110). However, somatic mtDNA deletions were shown to be slightly higher in the SN of PD patients compared with age-matched controls (107). These observations demonstrated that mtDNA deletions are specific to nigral neurons, which may increase their susceptibility to oxidative stress and hence contribute to their selective loss in PD. mtDNA is vulnerable to oxidative damage compared with nuclear DNA, since mitochondria are the main sites of ROS production and lack protective histones $(103,111)$. mtDNA damage is associated with the instability of genes and proteins, defect in the ETC and increased ROS production, leading to compromised bioenergetic functions and cell damage. A number of mitochondria-related diseases could be linked to mutations of the mitochondrial genome (104,106-108).

mtDNA has an autonomously replicating genome encoding a spectrum of mitochondrial respiratory chain proteins (112), and their deletions result in mitochondrial respiratory chain dysfunction (107). The ability to synthesize and repair mitochondrial genomes (mtDNA) is required for the mitochondria to maintain their bioactivities, which rely mainly on mtDNA polymerase. The polymerase $\gamma 1$ (POLG1) enzyme is a nuclear-encoded gene product that plays an important role in polymerase synthesis and mtDNA maintenance (113). Mutations in POLG1 have been shown to be associated with severe progressive multisystem disorders, including PD, supporting the involvement of the defective mtDNA in dopaminergic neuron degeneration (114-116). POLG1 mutations contribute to a gradual accumulation of secondary deletions in mtDNA, resulting in dysfunction of the respiratory chain (117). Recently, several studies have shown that selective increased mtDNA damage marker abasic site levels could be detected in the $\mathrm{SN}$ in postmortem brains of PD patients. Abasic sites were also shown in brain tissues from mice treated with neuronal toxins rotenone to induce PD-like pathology (118). Abasic sites are segments of DNA that have lost either a purine or a pyrimidine base, resulting in blockage of the polymerase during DNA replication and transcription (119). These studies demonstrated that dopaminergic neuronal injury could be ascribed to mtDNA damage, leading to ETC inhibition and mitochondrial dysfunction in PD pathogenesis. Therefore, the inhibition of this mtDNA damage may be beneficial for PD in neurodegenerative conditions. 


\section{Abnormalities of mitochondrial fusion and fission in PD}

Mitochondria are dynamic organelles with constant changes of morphology that are regulated by mitochondrial fission and fusion. The dynamic balance between fission and fusion is essential for the normal function of the mitochondria and plays a vital role in cellular bioactivities $(120,121)$. Mitochondrial fission serves as a mechanism to facilitate the equal segregation of the mitochondria into daughter cells in mitochondrial division, and to target damaged segments of the mitochondria in the autophagic process. This process is controlled by dynamin-related protein 1 (Drp1), a cytoplasmic dynamin GTPase that translocates to the mitochondria and locates to the OMM in response to mitochondrial dysfunction $(122,123)$. Cytosolic $\mathrm{Ca}^{2+}$ is a key mediator in its translocation via the activation of calcineurin and the dephosphorylation of Ser-637 residue in Drp1 (124). Ser-637 of Drp1 can also be phosphorylated by calmodulin kinase I $\alpha$, leading to its mitochondrial translocation (125). The translocated Drp1 provides a structural device for mechanical force in physical excision of the membrane by formatting a multimeric complex around the OMM (126). Mitochondrial fission 1 protein (Fis1) is another important regulator protein involved in mitochondrial fission. Overexpression of Fis1 has been described to activate the fission process, and results in mitochondrial fragmentation (127). This protein can also trigger autophagy to remove damaged mitochondria, thus contributing to the maintenance of cellular functions (128).

Mitochondrial fusion is another mechanism to maintain mitochondrial bioactivities by mixing the contents of mitochondria, thus enabling its protein complementation, mtDNA repair and equal distribution of metabolites (129). This process depends on three GTPase proteins: Mitofusin 1 (Mfn1), Mfn2 and optic atrophy protein 1 (OPA1) $(130,131)$. Mfn1 and Mfn2 are mitochondrial transmembrane proteins localized to the OMM, and they mediate the outer-membrane connection of neighboring mitochondria by forming homo-oligomeric and hetero-oligomeric complexes via interaction with their coiled-coil domains (132-134). OPA1 is an inner membrane protein mediating IMM fusion. Mitochondrial membrane potential is essential for the connection of the OMM and the IMM, and for maintenance of the mitochondrial tubular network $(135,136)$. Defects in the ETC complexes and damage to the mitochondrial membrane potential contribute to mitochondrial network disintegration, leading to increased fragmentation and cell death (137). Recent studies have suggested that defects in mitochondrial fission and fusion may be one of the underlying mechanisms responsible for mitochondria-mediated neurodegenerative disease (13). Mitochondrial fission and fragmentation can be observed in PD cellular models induced by the neurotoxins rotenone and 1-methyl-4-phenylpyridinium in a rat dopaminergic cell line (138). Depolarization of the mitochondrial membrane causes the loss of OPA1 and the MFNs, leading to the inhibition of fusion and mitochondrial fragmentation (139). The discharge of membrane potential is considered to be a critical factor associated with mitochondrial dysfunction and cell death in neurodegenerative diseases $(44,140)$, indicating its involvement as one of the mechanisms responsible for the degeneration of dopaminergic neurons in PD pathogenesis.
Recent studies have linked Parkin and the PTEN-induced putative kinase 1 (PINK1)/Parkin pathway with the maintenance of mitochondrial dynamics $(141,142)$. PINK1 is a mitochondrial kinase and can be translocated to the OMM where it is cleaved rapidly by presenilin-associated rhomboid-like protease (143). However, when mitochondria are impaired and deplete of membrane potential, the protease activity is inhibited, with the subsequent accumulation of PINK1 on the mitochondrial membrane $(143,144)$. The elevated levels of PINK1 on the OMM trigger the translocation of Parkin to the mitochondria by phosphorylating the protein at Thr-175 in a kinase activity-dependent manner $(145,146)$. PINK1/Parkin mediate mitochondrial fusion and fission dependent on dynamin-like GTPases, including Mfn1, Mfn2, OPA1 and Fis1, and the potential mechanisms of the regulation have been well documented (6). Parkin can facilitate sequestration and elimination of damaged mitochondria via mitophagy, a protective mechanism for maintaining the recycling of proteins and mitochondrial homeostasis to limit cell death (147). Together, the aforementioned studies may provide a molecular target to alleviate the severity of mitochondria-mediated dopaminergic neuron damage in PD pathogenesis.

\section{Conclusion}

PD is characterized by the progressive degeneration and death of dopaminergic neurons in the SN. The development of molecular and cellular mechanisms associated with dopaminergic neuron damage has implicated the involvement of a range of events in PD pathogenesis, through distinct and divert pathways, to cause progressive neuron damage. Although the precise mechanisms of neuronal damage remain unclear, abnormalities of the mitochondria appear to be a converging point in the cell death processes. Understanding the mechanisms underlying mitochondria-mediated neuron death may provide a promising management solution for PD treatment, however, this requires further elucidation.

\section{References}

1. Forno LS: Neuropathology of Parkinson's disease. J Neuropathol Exp Neurol 55: 259-272, 1996.

2. Martin LJ: Biology of mitochondria in neurodegenerative diseases. Prog Mol Biol Transl Sci 107: 355-415, 2012.

3. Trancikova A, Tsika E and Moore DJ: Mitochondrial dysfunction in genetic animal models of Parkinson's disease. Antioxid Redox Signal 16: 896-919, 2012.

4. Ryan BJ, Hoek S, Fon EA and Wade-Martins R: Mitochondrial dysfunction and mitophagy in Parkinson's: From familial to sporadic disease. Trends Biochem Sci 40: 200-210, 2015.

5. Moon HE and Paek SH: Mitochondrial dysfunction in Parkinson's disease. Exp Neurobiol 24: 103-116, 2015.

6. Exner N, Lutz AK, Haass C and Winklhofer KF: Mitochondrial dysfunction in Parkinson's disease: Molecular mechanisms and pathophysiological consequences. EMBO J 31: 3038-3062, 2012.

7. Mounsey RB and Teismann P: Mitochondrial dysfunction in Parkinson's disease: Pathogenesis and neuroprotection. Parkinsons Dis 2010: 617472, 2011.

8. Martin LJ: Mitochondrial and cell death mechanisms in neurodegenerative diseases. Pharmaceuticals (Basel) 3: 839-915, 2010.

9. Reddy PH and Reddy TP: Mitochondria as a therapeutic target for aging and neurodegenerative diseases. Curr Alzheimer Res 8: 393-409, 2011.

10. Reddy PH and Beal MF: Are mitochondria critical in the pathogenesis of Alzheimer's disease? Brain Res Brain Res Rev 49: 618-632, 2005.

11. Rostovtseva TK, Tan W and Colombini M: On the role of VDAC in apoptosis: fact and fiction. J Bioenerg Biomembr 37: 129-142, 2005. 
12. Okada SF, O'Neal WK, Huang P, Nicholas RA, Ostrowski LE, Craigen WJ, Lazarowski ER and Boucher RC: Voltage-dependent anion channel-1 (VDAC-1) contributes to ATP release and cell volume regulation in murine cells. J Gen Physiol 124: 513-526, 2004.

13. Camara AK, Lesnefsky EJ and Stowe DF: Potential therapeutic benefits of strategies directed to mitochondria. Antioxid Redox Signal 13: 279-347, 2010.

14. Bernardi P: Mitochondrial transport of cations: Channels, exchangers, and permeability transition. Physiol Rev 79: $1127-1155,1999$.

15. Teshima Y, Akao M, Jones SP and Marbán E: Uncoupling protein-2 overexpression inhibits mitochondrial death pathway in cardiomyocytes. Circ Res 93: 192-200, 2003.

16. O'Rourke B: Mitochondrial ion channels. Annu Rev Physiol 69: 19-49, 2007

17. Wingrove DE and Gunter TE: Kinetics of mitochondrial calcium transport. II. A kinetic description of the sodium-dependent calcium efflux mechanism of liver mitochondria and inhibition by ruthenium red and by tetraphenylphosphonium. J Biol Chem 261: 15166-15171, 1986.

18. Bernardi P, Krauskopf A, Basso E, Petronilli V, Blachly-Dyson E, Di Lisa $\mathrm{F}$ and Forte MA: The mitochondrial permeability transition from in vitro artifact to disease target. FEBS J 273 : 2077-2099, 2006.

19. Leung AW and Halestrap AP: Recent progress in elucidating the molecular mechanism of the mitochondrial permeability transition pore. Biochim Biophys Acta 1777: 946-952, 2008

20. Vyssokikh MY, Katz A, Rueck A, Wuensch C, Dörner A Zorov DB and Brdiczka D: Adenine nucleotide translocator isoforms 1 and 2 are differently distributed in the mitochondrial inner membrane and have distinct affinities to cyclophilin D. Biochem J 358: 349-358, 2001.

21. Halestrap AP and Brenner C: The adenine nucleotide translocase: A central component of the mitochondrial permeability transition pore and key player in cell death. Curr Med Chem 10: $1507-1525,2003$

22. Ojala D, Montoya J and Attardi G: tRNA punctuation model of RNA processing in human mitochondria. Nature 290: 470-474, 1981.

23. Reddy PH: Amyloid precursor protein-mediated free radicals and oxidative damage: Implications for the development and progression of Alzheimer's disease. J Neurochem 96: 1-13, 2006.

24. Brookes PS, Levonen AL, Shiva S, Sarti P and Darley-Usmar VM Mitochondria: Regulators of signal transduction by reactive oxygen and nitrogen species. Free Radic Biol Med 33: 755-764, 2002

25. Dröge W: Free radicals in the physiological control of cell function. Physiol Rev 82: 47-95, 2002

26. Kudin AP, Bimpong-Buta NY, Vielhaber S, Elger CE and Kunz WS: Characterization of superoxide-producing sites in isolated brain mitochondria. J Biol Chem 279: 4127-4135, 2004

27. Brand MD, Affourtit C, Esteves TC, Green K, Lambert AJ Miwa S, Pakay JL and Parker N: Mitochondrial superoxide: Production, biological effects, and activation of uncoupling proteins. Free Radic Biol Med 37: 755-767, 2004.

28. Andreyev AY, Kushnareva YE and Starkov AA: Mitochondrial metabolism of reactive oxygen species. Biochemistry (Mosc) 70: 200-214, 2005

29. Cadenas E and Davies KJ: Mitochondrial free radical generation, oxidative stress, and aging. Free Radic Biol Med 29: 222-230, 2000.

30. Kudin AP, Debska-Vielhaber G and Kunz WS: Characterization of superoxide production sites in isolated rat brain and skeletal muscle mitochondria. Biomed Pharmacother 59: 163-168, 2005.

31. Kussmaul L and Hirst J: The mechanism of superoxide production by NADH:ubiquinone oxidoreductase (complex I) from bovine heart mitochondria. Proc Natl Acad Sci USA 103: 7607-7612, 2006

32. Rush JD and Koppenol WH: Oxidizing intermediates in the reaction of ferrous EDTA with hydrogen peroxide. Reactions with organic molecules and ferrocytochrome c. J Biol Chem 261: 6730-6733, 1986.

33. Antunes F, Han D and Cadenas E: Relative contributions of heart mitochondria glutathione peroxidase and catalase to $\mathrm{H}(2) \mathrm{O}(2)$ detoxification in in vivo conditions. Free Radic Biol Med 33 1260-1267, 2002.

34. Morán M, Moreno-Lastres D, Marín-Buera L, Arenas J, Martín MA and Ugalde C: Mitochondrial respiratory chain dysfunction: Implications in neurodegeneration. Free Radic Biol Med 53: 595-609, 2012
35. Gutteridge JM: Superoxide-dependent formation of hydroxyl radicals from ferric-complexes and hydrogen peroxide: An evaluation of fourteen iron chelators. Free Radic Res Commun 9: $119-125,1990$

36. Hwang O: Role of oxidative stress in Parkinson's disease. Exp Neurobiol 22: 11-17, 2013.

37. Dias V, Junn E and Mouradian MM: The role of oxidative stress in Parkinson's disease. J Parkinsons Dis 3: 461-491, 2013.

38. Jenner P: Oxidative stress in Parkinson's disease. Ann Neurol 53 (Suppl 3): S26-S38, 2003.

39. Alam ZI, Jenner A, Daniel SE, Lees AJ, Cairns N, Marsden CD, Jenner P and Halliwell B: Oxidative DNA damage in the parkinsonian brain: An apparent selective increase in 8-hydroxyguanine levels in substantia nigra. J Neurochem 69: 1196-1203, 1997.

40. Yoritaka A, Hattori N, Uchida K, Tanaka M, Stadtman ER and Mizuno Y: Immunohistochemical detection of 4-hydroxynonenal protein adducts in Parkinson disease. Proc Natl Acad Sci USA 93: 2696-2701, 1996.

41. Li DW, Yao M, Dong YH, Tang MN, Chen W, Li GR and Sun BQ: Guanosine exerts neuroprotective effects by reversing mitochondrial dysfunction in a cellular model of Parkinson's disease. Int J Mol Med 34: 1358-1364, 2014.

42. Seet RC, Lee CY, Lim EC, Tan JJ, Quek AM, Chong WL, Looi WF, Huang SH, Wang $\mathrm{H}$ and Chan YH: Oxidative damage in Parkinson disease: Measurement using accurate biomarkers. Free Radic Biol Med 48: 560-566, 2010.

43. Callio J, Oury TD and Chu CT: Manganese superoxide dismutase protects against 6-hydroxydopamine injury in mouse brains. J Biol Chem 280: 18536-18542, 2005.

44. Vila M and Przedborski S: Targeting programmed cell death in neurodegenerative diseases. Nat Rev Neurosci 4: 365-375, 2003.

45. Perier C, Bové J, Vila M and Przedborski S: The rotenone model of Parkinson's disease. Trends Neurosci 26: 345-346, 2003.

46. Sun SY, An CN and Pu XP: DJ-1 protein protects dopaminergic neurons against 6-OHDA/MG-132-induced neurotoxicity in rats. Brain Res Bull 88: 609-616, 2012.

47. Heikkila RE, Hess A and Duvoisin RC: Dopaminergic neurotoxicity of 1-methyl-4-phenyl-1,2,5,6-tetrahydropyridine in mice. Science 224: 1451-1453, 1984

48. Barzilai A and Yamamoto K: DNA damage responses to oxidative stress. DNA Repair (Amst) 3: 1109-1115, 2004.

49. Ruipérez V, Darios F and Davletov B: Alpha-synuclein, lipids and Parkinson's disease. Prog Lipid Res 49: 420-428, 2010.

50. Mariani E, Polidori MC, Cherubini A and Mecocci P: Oxidative stress in brain aging, neurodegenerative and vascular diseases: An overview. J Chromatogr B Analyt Technol Biomed Life Sci 827: 65-75, 2005.

51. Montine TJ, Neely MD, Quinn JF, Beal MF, Markesbery WR, Roberts LJ II and Morrow JD: Lipid peroxidation in aging brain and Alzheimer's disease. Free Radic Biol Med 33: 620-626, 2002

52. Liu W, Kato M, Akhand AA, Hayakawa A, Suzuki H, Miyata T, Kurokawa K, Hotta Y, Ishikawa N and Nakashima I: 4-hydroxynonenal induces a cellular redox status-related activation of the caspase cascade for apoptotic cell death. J Cell Sci 113: 635-641, 2000

53. Schmidt H, Grune T, Müller R, Siems WG and Wauer RR: Increased levels of lipid peroxidation products malondialdehyde and 4-hydroxynonenal after perinatal hypoxia. Pediatr Res 40: 15-20, 1996.

54. Montine KS, Quinn JF, Zhang J, Fessel JP, Roberts LJ II, Morrow JD and Montine TJ: Isoprostanes and related products of lipid peroxidation in neurodegenerative diseases. Chem Phys Lipids 128: 117-124, 2004.

55. Lotharius $\mathbf{J}$ and Brundin P: Pathogenesis of Parkinson's disease: Dopamine, vesicles and alpha-synuclein. Nat Rev Neurosci 3: 932-942, 2002

56. Fornstedt B and Carlsson A: A marked rise in 5-S-cysteinyl-dopamine levels in guinea-pig striatum following reserpine treatment. J Neural Transm 76: 155-161, 1989.

57. Youdim MB, Edmondson D and Tipton KF: The therapeutic potential of monoamine oxidase inhibitors. Nat Rev Neurosci 7: 295-309, 2006.

58. Fowler JS, Volkow ND, Wang GJ, Logan J, Pappas N, Shea C and MacGregor R: Age-related increases in brain monoamine oxidase B in living healthy human subjects. Neurobiol Aging 18: 431-435, 1997.

59. Nagatsu $T$ and Sawada M: Molecular mechanism of the relation of monoamine oxidase B and its inhibitors to Parkinson's disease: Possible implications of glial cells. J Neural Transm Suppl 71: 53-65, 2006 
60. Kumar MJ and Andersen JK: Perspectives on MAO-B in aging and neurological disease: Where do we go from here? Mol Neurobiol 30: 77-89, 2004.

61.Norris EH, Giasson BI, Hodara R, Xu S, Trojanowski JQ, Ischiropoulos $\mathrm{H}$ and Lee VM: Reversible inhibition of alpha-synuclein fibrillization by dopaminochrome-mediated conformational alterations. J Biol Chem 280: 21212-21219, 2005.

62.Zecca L, Wilms H, Geick S, Claasen JH, Brandenburg LO, Holzknecht C, Panizza ML, Zucca FA, Deuschl G, Sievers J, et al: Human neuromelanin induces neuroinflammation and neurodegeneration in the rat substantia nigra: Implications for Parkinson's disease. Acta Neuropathol 116: 47-55, 2008.

63. Jomova $\mathrm{K}$ and Valko M: Advances in metal-induced oxidative stress and human disease. Toxicology 283: 65-87, 2011

64. Núñez MT, Urrutia P, Mena N, Aguirre P, Tapia V and Salazar J: Iron toxicity in neurodegeneration. Biometals 25: 761-776, 2012

65. Sadrzadeh SM and Saffari Y: Iron and brain disorders. Am J Clin Pathol 121 (Suppl): S64-S70, 2004.

66. Sian-Hülsmann J, Mandel S, Youdim MB and Riederer P: The relevance of iron in the pathogenesis of Parkinson's disease. J Neurochem 118: 939-957, 2011.

67. Sziráki I, Mohanakumar KP, Rauhala P, Kim HG, Yeh KJ and Chiueh CC: Manganese: A transition metal protects nigrostriatal neurons from oxidative stress in the iron-induced animal model of parkinsonism. Neuroscience 85: 1101-1111, 1998.

68. Lan J and Jiang DH: Desferrioxamine and vitamin E protect against iron and MPTP-induced neurodegeneration in mice. J Neural Transm Vienna 104: 469-481, 1997.

69. Faucheux BA, Martin ME, Beaumont C, Hauw JJ, Agid Y and Hirsch EC: Neuromelanin associated redox-active iron is increased in the substantia nigra of patients with Parkinson's disease. J Neurochem 86: 1142-1148, 2003.

70. Yokoyama H, Kuroiwa H, Yano R and Araki T: Targeting reactive oxygen species, reactive nitrogen species and inflammation in MPTP neurotoxicity and Parkinson's disease. Neurol Sci 29 293-301, 2008.

71. Duchen MR: Mitochondria in health and disease: Perspectives on a new mitochondrial biology. Mol Aspects Med 25: 365-451, 2004

72. Henchcliffe C and Beal MF: Mitochondrial biology and oxidative stress in Parkinson disease pathogenesis. Nat Clin Pract Neurol 4: 600-609, 2008.

73. Mythri RB, Jagatha B, Pradhan N, Andersen J and Bharath MM: Mitochondrial complex I inhibition in Parkinson's disease: How can curcumin protect mitochondria? Antioxid Redox Signal 9: 399-408, 2007.

74. Adams JM and Cory S: The Bcl-2 protein family: Arbiters of cell survival. Science 281: 1322-1326, 1998.

75. Crompton M: The mitochondrial permeability transition pore and its role in cell death. Biochem J 341: 233-249, 1999.

76. Burchell VS, Gandhi S, Deas E, Wood NW, Abramov AY and Plun-Favreau H: Targeting mitochondrial dysfunction in neurodegenerative disease: Part I. Expert Opin Ther Targets 14: $369-385,2010$.

77. Moon Y, Lee KH, Park JH, Geum D and Kim K: Mitochondrial membrane depolarization and the selective death of dopaminergic neurons by rotenone: Protective effect of coenzyme Q10. J Neurochem 93: 1199-1208, 2005.

78. McCarthy S, Somayajulu M, Sikorska M, Borowy-Borowski H and Pandey S: Paraquat induces oxidative stress and neuronal cell death; neuroprotection by water-soluble Coenzyme Q10. Toxicol Appl Pharmacol 201: 21-31, 2004.

79. Cleren C, Yang L, Lorenzo B, Calingasan NY, Schomer A, Sireci A, Wille EJ and Beal MF: Therapeutic effects of coenzyme Q10 (CoQ10) and reduced CoQ10 in the MPTP model of Parkinsonism. J Neurochem 104: 1613-1621, 2008.

80.Dubois C, Prevarskaya N and Vanden Abeele F: The calcium-signaling toolkit: Updates needed. Biochim Biophys Acta 1863: 1337-1343, 2016

81. Santo-Domingo J, Wiederkehr A and De Marchi U: Modulation of the matrix redox signaling by mitochondrial $\mathrm{Ca}(2)$. World $\mathrm{J}$ Biol Chem 6: 310-323, 2015

82. Nicholls DG: Mitochondrial function and dysfunction in the cell: Its relevance to aging and aging-related disease. Int J Biochem Cell Biol 34: 1372-1381, 2002.

83. Kirichok Y, Krapivinsky G and Clapham DE: The mitochondrial calcium uniporter is a highly selective ion channel. Nature 427: 360-364, 2004.
84. Gincel D, Zaid H and Shoshan-Barmatz V: Calcium binding and translocation by the voltage-dependent anion channel: A possible regulatory mechanism in mitochondrial function. Biochem J 358: 147-155, 2001.

85. Takeuchi A, Kim B and Matsuoka S: The destiny of $\mathrm{Ca}(2+)$ released by mitochondria. J Physiol Sci 65: 11-24, 2015.

86. Plovanich M, Bogorad RL, Sancak Y, Kamer KJ, Strittmatter L, Li AA, Girgis HS, Kuchimanchi S, De Groot J, Speciner L, et al: MICU2, a paralog of MICU1, resides within the mitochondrial uniporter complex to regulate calcium handling. PLoS One 8: e55785, 2013.

87.Perocchi F, Gohil VM, Girgis HS, Bao XR, McCombs JE, Palmer AE and Mootha VK: MICU1 encodes a mitochondrial $\mathrm{EF}$ hand protein required for $\mathrm{Ca}(2+)$ uptake. Nature 467: 291-296, 2010.

88. McCormack JG and Denton RM: Mitochondrial $\mathrm{Ca}^{2+}$ transport and the role of intramitochondrial $\mathrm{Ca}^{2+}$ in the regulation of energy metabolism. Dev Neurosci 15: 165-173, 1993.

89. Balaban RS: Cardiac energy metabolism homeostasis: Role of cytosolic calcium. J Mol Cell Cardiol 34: 1259-1271, 2002.

90. Alderton WK, Cooper CE and Knowles RG: Nitric oxide synthases: Structure, function and inhibition. Biochem J 357: 593-615, 2001

91. Jekabsone A, Ivanoviene L, Brown GC and Borutaite V: Nitric oxide and calcium together inactivate mitochondrial complex I and induce cytochrome $c$ release. J Mol Cell Cardiol 35: 803-809, 2003.

92. Brookes PS, Yoon Y, Robotham JL, Anders MW and Sheu SS: Calcium, ATP, and ROS: A mitochondrial love-hate triangle. Am J Physiol Cell Physiol 287: C817-C833, 2004.

93. Muravchick S and Levy RJ: Clinical implications of mitochondrial dysfunction. Anesthesiology 105: 819-837, 2006.

94. O'Rourke B: Pathophysiological and protective roles of mitochondrial ion channels. J Physiol 529: 23-36, 2000.

95. Di Lisa F and Bernardi P: A CaPful of mechanisms regulating the mitochondrial permeability transition. J Mol Cell Cardiol 46: 775-780, 2009

96. Jones SP, Teshima Y, Akao M and Marbán E: Simvastatin attenuates oxidant-induced mitochondrial dysfunction in cardiac myocytes. Circ Res 93: 697-699, 2003.

97. Celardo I, Martins LM and Gandhi S: Unravelling mitochondrial pathways to Parkinson's disease. Br J Pharmacol 171: 1943-1957, 2014.

98. Surmeier DJ, Guzman JN, Sanchez-Padilla J and Goldberg JA The origins of oxidant stress in Parkinson's disease and therapeutic strategies. Antioxid Redox Signal 14: 1289-1301, 2011.

99. Perier C, Tieu K, Guégan C, Caspersen C, Jackson-Lewis V, Carelli V, Martinuzzi A, Hirano M, Przedborski S and Vila M: Complex I deficiency primes Bax-dependent neuronal apoptosis through mitochondrial oxidative damage. Proc Natl Acad Sci USA 102: 19126-19131, 2005.

100. Boatright KM and Salvesen GS: Mechanisms of caspase activation. Curr Opin Cell Biol 15: 725-731, 2003.

101.Kumar S: Caspase function in programmed cell death. Cell Death Differ 14: 32-43, 2007.

102. Javadov S, Choi A, Rajapurohitam V, Zeidan A, Basnakian AG and Karmazyn M: NHE-1 inhibition-induced cardioprotection against ischaemia/reperfusion is associated with attenuation of the mitochondrial permeability transition. Cardiovasc Res 77: 416-424, 2008.

103. Wallace DC: A mitochondrial paradigm of metabolic and degenerative diseases, aging, and cancer: A dawn for evolutionary medicine. Annu Rev Genet 39: 359-407, 2005.

104. Yang JL, Weissman L, Bohr VA and Mattson MP: Mitochondrial DNA damage and repair in neurodegenerative disorders. DNA Repair (Amst) 7: 1110-1120, 2008

105.Levy RJ and Deutschman CS: Deficient mitochondrial biogenesis in critical illness: Cause, effect, or epiphenomenon? Crit Care 11: 158, 2007.

106. Elstner M, Müller SK, Leidolt L, Laub C, Krieg L, Schlaudraff F, Liss B, Morris C, Turnbull DM, Masliah E, et al: Neuromelanin, neurotransmitter status and brainstem location determine the differential vulnerability of catecholaminergic neurons to mitochondrial DNA deletions. Mol Brain 4: 43, 2011

107. Kraytsberg Y, Kudryavtseva E, McKee AC, Geula C, Kowall NW and Khrapko K: Mitochondrial DNA deletions are abundant and cause functional impairment in aged human substantia nigra neurons. Nat Genet 38: 518-520, 2006. 
108. Bender A, Krishnan KJ, Morris CM, Taylor GA, Reeve AK, Perry RH, Jaros E, Hersheson JS, Betts J, Klopstock T, et al: High levels of mitochondrial DNA deletions in substantia nigra neurons in aging and Parkinson disease. Nat Genet 38: 515-517, 2006.

109. Ekstrand MI, Terzioglu M, Galter D, Zhu S, Hofstetter C, Lindqvist E, Thams S, Bergstrand A, Hansson FS, Trifunovic A, et al: Progressive parkinsonism in mice with respiratory-chain-deficient dopamine neurons. Proc Natl Acad Sci USA 104: 1325-1330, 2007.

110. Tanner CM, Kamel F, Ross GW, Hoppin JA, Goldman SM, Korell M, Marras C, Bhudhikanok GS, Kasten M, Chade AR, et al: Rotenone, paraquat, and Parkinson's disease. Environ Health Perspect 119: 866-872, 2011.

111. Halliwell B: Role of free radicals in the neurodegenerative diseases: Therapeutic implications for antioxidant treatment. Drugs Aging 18: 685-716, 2001.

112. Reeve AK, Krishnan KJ and Turnbull D: Mitochondrial DNA mutations in disease, aging, and neurodegeneration. Ann NY Acad Sci 1147: 21-29, 2008.

113. Ropp PA and Copeland WC: Cloning and characterization of the human mitochondrial DNA polymerase, DNA polymerase gamma. Genomics 36: 449-458, 1996.

114. Luoma P, Melberg A, Rinne JO, Kaukonen JA, Nupponen NN, Chalmers RM, Oldfors A, Rautakorpi I, Peltonen L, Majamaa K, et al: Parkinsonism, premature menopause, and mitochondrial DNA polymerase gamma mutations: Clinical and molecular genetic study. Lancet 364: 875-882, 2004.

115. Wong LJ, Naviaux RK, Brunetti-Pierri N, Zhang Q, Schmitt ES, Truong C, Milone M, Cohen BH, Wical B, Ganesh J, et al: Molecular and clinical genetics of mitochondrial diseases due to POLG mutations. Hum Mutat 29: E150-E172, 2008.

116. Gui YX, Xu ZP, Lv W, Zhao JJ and Hu XY: Evidence for polymerase gamma, POLG1 variation in reduced mitochondrial DNA copy number in Parkinson's disease. Parkinsonism Relat Disord 21: 282-286, 2015.

117. Hudson G and Chinnery PF: Mitochondrial DNA polymerase-gamma and human disease. Hum Mol Genet 15: R244-R252, 2006.

118. Sanders LH, McCoy J, Hu X, Mastroberardino PG, Dickinson BC, Chang CJ, Chu CT, Van Houten B and Greenamyre JT: Mitochondrial DNA damage: Molecular marker of vulnerable nigral neurons in Parkinson's disease. Neurobiol Dis 70: 214-223, 2014.

119. Wilson DM III and Barsky D: The major human abasic endonuclease: Formation, consequences and repair of abasic lesions in DNA. Mutat Res 485: 283-307, 2001.

120. Benard G and Karbowski M: Mitochondrial fusion and division: Regulation and role in cell viability. Semin Cell Dev Biol 20: 365-374, 2009.

121. Soubannier V and McBride HM: Positioning mitochondrial plasticity within cellular signaling cascades. Biochim Biophys Acta 1793: 154-170, 2009.

122. Schrader M: Shared components of mitochondrial and peroxisomal division. Biochim Biophys Acta 1763: 531-541, 2006.

123. Ishihara N, Jofuku A, Eura Y and Mihara K: Regulation of mitochondrial morphology by membrane potential, and DRP1-dependent division and FZO1-dependent fusion reaction in mammalian cells. Biochem Biophys Res Commun 301: 891-898, 2003.

124. Cereghetti GM, Stangherlin A, Martins de Brito O, Chang CR, Blackstone C, Bernardi P and Scorrano L: Dephosphorylation by calcineurin regulates translocation of Drp1 to mitochondria. Proc Natl Acad Sci USA 105: 15803-15808, 2008.

125. Han XJ, Lu YF, Li SA, Kaitsuka T, Sato Y, Tomizawa K, Nairn AC, Takei K, Matsui H and Matsushita M: CaM kinase I alpha-induced phosphorylation of Drpl regulates mitochondrial morphology. J Cell Biol 182: 573-585, 2008.

126. Reddy PH, Reddy TP, Manczak M, Calkins MJ, Shirendeb U and Mao P: Dynamin-related protein 1 and mitochondrial fragmentation in neurodegenerative diseases. Brain Res Brain Res Rev 67: 103-118, 2011.

127. James DI, Parone PA, Mattenberger Y and Martinou JC: hFis1, a novel component of the mammalian mitochondrial fission machinery. J Biol Chem 278: 36373-36379, 2003.
128. Gomes LC and Scorrano L: High levels of Fis1, a pro-fission mitochondrial protein, trigger autophagy. Biochim Biophys Acta 1777: 860-866, 2008.

129. Santos D and Cardoso SM: Mitochondrial dynamics and neuronal fate in Parkinson's disease. Mitochondrion 12 428-437, 2012

130. Reddy PH: Amyloid beta, mitochondrial structural and functional dynamics in Alzheimer's disease. Exp Neurol 218: 286-292, 2009.

131. Reddy PH: Mitochondrial dysfunction in aging and Alzheimer's disease: Strategies to protect neurons. Antioxid Redox Signal 9: 1647-1658, 2007.

132. Chen H, Chomyn A and Chan DC: Disruption of fusion results in mitochondrial heterogeneity and dysfunction. J Biol Chem 280: 26185-26192, 2005.

133. Ishihara N, Eura Y and Mihara K: Mitofusin 1 and 2 play distinct roles in mitochondrial fusion reactions via GTPase activity. J Cell Sci 117: 6535-6546, 2004.

134.Züchner S, Mersiyanova IV, Muglia M, Bissar-Tadmouri N, Rochelle J, Dadali EL, Zappia M, Nelis E, Patitucci A, Senderek J, et al: Mutations in the mitochondrial GTPase mitofusin 2 cause Charcot-Marie-Tooth neuropathy type $2 \mathrm{~A}$. Nat Genet 36: 449-451, 2004

135. Armstrong JS: Mitochondria-directed therapeutics. Antioxid Redox Signal 10: 575-578, 2008

136. Chan DC: Mitochondria: Dynamic organelles in disease, aging, and development. Cell 125: 1241-1252, 2006

137. McBride HM, Neuspiel M and Wasiak S: Mitochondria: More than just a powerhouse. Curr Biol 16: R551-R560, 2006.

138. Barsoum MJ, Yuan H, Gerencser AA, Liot G, Kushnareva Y, Gräber S, Kovacs I, Lee WD, Waggoner J, Cui J, et al: Nitric oxide-induced mitochondrial fission is regulated by dynamin-related GTPases in neurons. EMBO J 25: 3900-3911, 2006

139.Head B, Griparic L, Amiri M, Gandre-Babbe S and van der Bliek AM: Inducible proteolytic inactivation of OPA1 mediated by the OMA1 protease in mammalian cells. J Cell Biol 187: 959-966, 2009.

140. Abou-Sleiman PM, Muqit MM and Wood NW: Expanding insights of mitochondrial dysfunction in Parkinson's disease. Nat Rev Neurosci 7: 207-219, 2006.

141. Yang Y, Gehrke S, Imai Y, Huang Z, Ouyang Y, Wang JW, Yang L, Beal MF, Vogel H and Lu B: Mitochondrial pathology and muscle and dopaminergic neuron degeneration caused by inactivation of Drosophila Pink1 is rescued by Parkin. Proc Natl Acad Sci USA 103: 10793-10798, 2006.

142.Park J, Lee SB, Lee S, Kim Y, Song S, Kim S, Bae E, Kim J, Shong M, Kim JM and Chung J: Mitochondrial dysfunction in Drosophila PINK1 mutants is complemented by parkin. Nature 441: 1157-1161, 2006.

143. Jin SM, Lazarou M, Wang C, Kane LA, Narendra DP and Youle RJ: Mitochondrial membrane potential regulates PINK1 import and proteolytic destabilization by PARL. J Cell Biol 191: 933-942, 2010

144. Narendra DP, Jin SM, Tanaka A, Suen DF, Gautier CA, Shen J, Cookson MR and Youle RJ: PINK1 is selectively stabilized on impaired mitochondria to activate Parkin. PLoS Biol 8: e1000298,2010.

145.Kim Y, Park J, Kim S, Song S, Kwon SK, Lee SH, Kitada T, Kim JM and Chung J: PINK1 controls mitochondrial localization of Parkin through direct phosphorylation. Biochem Biophys Res Commun 377: 975-980, 2008.

146. Sha D, Chin LS and Li L: Phosphorylation of parkin by Parkinson disease-linked kinase PINK1 activates parkin E3 ligase function and NF-kappaB signaling. Hum Mol Genet 19: 352-363, 2010.

147. Geisler S, Holmström KM, Skujat D, Fiesel FC, Rothfuss OC, Kahle PJ and Springer W: PINK1/Parkin-mediated mitophagy is dependent on VDAC1 and p62/SQSTM1. Nat Cell Biol 12: $119-131,2010$ 